\title{
Architecture - Behaviour - Properties Relationship in Star-Shaped MPA-PMMA and MPA-PS Hyper- Branched Copolymers
}

\author{
Gabriel Ríos Valer, Gisela Díaz, Juan M Giussi, Marcelo Ceolín \\ Instituto de Investigaciones Fisicoquímicas Teóricas y Aplicadas (INIFTA) - Departamento de Química - Facultad de \\ Ciencias Exactas - Universidad Nacional de La Plata - CONICET, 1900 La Plata - Argentina \\ *Corresponding author: E-mail: jmgiussi@ inifta.unlp.edu.ar
}

Received: 14 August 2018, Revised: 31 October 2018 and Accepted: 31 October 2018

DOI: 10.5185/amlett.2019.2224

www.vbripress.com/aml

\begin{abstract}
The molecular architecture of polymers is a crucial feature in the moment of think the relationship between properties and applications. The same polymer can present important differences according to its architecture and leads to different possible applications. In this paper, we describe the well preparation of hyperbranched copolymers based on bis (HydroxylMethyl) propionic acid polyester (MPA). The co-monomers introduced via atom transfer radical polymerization were methyl methacrylate (MMA) and styrene (St). In order to study the effect of confinement, linear PMMA and PSt have been prepared, and moreover different levels of branching of each polymer were prepared. The synthesised star PMPAPMMA and PMPA-PSt copolymers have been characterized and identified by infrared spectroscopy and nuclear magnetic resonance spectroscopy. Thermal transitions in solid state were studied using differential scanning calorimetry, and the thermal stability was evaluated by thermogravimetric analysis. Finally, solution properties have been evaluated thought Dynamic Light Scattering. Our results, obtained by a meticulous and systematic comparative study, showed a clear tendency between architectural level and thermal properties. Moreover, properties in solution revealed interesting response due to the modification of solvent nature. Copyright $($ C VBRI Press.
\end{abstract}

Keywords: Star-shaped hyperbranched copolymers with different generation levels, thermal characterization, solution properties.

\section{Introduction}

Branched polymers, including dendrimers and hyperbranched polymers, display important differences with regard to their linear counterparts. Branched entities contain high-density of localized polymer chains resulting in unique physical properties, such as high functionality and low viscosity as compared to their linear analogues with similar molecular weight opening a range of possibilities for application of these polymers in several areas of science and technology $[\mathbf{1 , 2}]$, such as coatings, resin formulations, additives, drug delivery, cosmetics, membranes, and lithography [3-6].

The difference between hyperbranched polymers and dendrimeric structures is their synthetic approach and specific microstructure. Dendrimers' microstructure is not probabilistic, and their synthesis is through a stepby-step sequence, each one with a reaction, isolation and purification part. Conversely, hyperbranched polymers are probabilistic systems and they are obtained in one step, indeed a simpler and more direct synthetic approach [7]. Nonetheless, the chemical-physical properties of these two types of macromolecules are considerably similar and hyperbranched polymers can perfectly replace dendrimers in many applications $[\mathbf{6 , 8} \mathbf{8}]$.

As far as the preparation of star-shaped hyperbranched polymers is concerned, numerous polymerization techniques have been used. Wan et al. [9, 10] combined atom transfer radical self-condensing vinyl polymerization and reversible additionfragmentation chain transfer polymerization (RAFT) to prepare hyperbranched polystyrene as core and poly (methyl methacrylate)-block-poly (ethylene glycol) methyl ether methacrylate (PMMA- $b$-PPEGMA) as brush shell. This system has provided excellent properties for use in lithium-ion batteries and ionic liquids. Alternatively, Pal et al.[11] prepared thermoand redox-responsive hyperbranched copolymers based on $\mathrm{N}$-isopropylacrylamide and $\mathrm{N}, \mathrm{N}$ '-bis (acryloyl) cystamine by RAFT.

On the other hand, over the past decade, numerous star-shaped polymers and copolymers have been prepared by atom transfer radical polymerization (ATRP) mainly within the Matyjaszewski Polymer Group [12]. This group used different strategies to prepare star-shaped hyperbranched polymers and some 
examples relevant to our work are explained below. Nese et al. observed that particle coupling depends on the number of arms and arm length in 10- and 20-arm starlike block copolymers based on poly (n-butyl acrylate) and poly (methyl methacrylate). These materials were synthesized by ATRP using short linear poly (2-bromoisobutyryloxyethyl acrylate) macroinitiators. Matyjaszewski also observed phase separation in these systems by atomic force microscopy and small-angle $\mathrm{X}$-ray scattering; and the mechanical and thermal properties of these copolymers were considerably different as compared to linear and star-shaped copolymers of low arm number with similar composition [13]. Li et al. [14] also reported the synthesis of amphiphilic star-shaped polymers based on poly (ethylene oxide)-b-polystyrene by ATRP to form crosslinkers woollen micelles. These star-shaped polymers showed low polydispersity and high molecular weight in highly diluted aqueous dispersions. Moreover, Gao et al. $[15,16]$ synthesized star-shaped copolymers with different microstructure and monomer sequences using different strategies based on ATRP. The resulting polymers confirmed this technique as a powerful strategy for the synthesis of various kinds of miktoarm star-shaped copolymers with high molecular weight and low polydispersity. Also based on Matyjaszewski work, Bencherif et al .[17] published the synthesis and evaluation of adhesion cells of poly (ethylene oxide) (PEO) star-shaped polymers. These star-shaped polymers obtained by ATRP have demonstrated that good polydispersity and cell interaction depend, to a certain extent, on polymer structure.

Among other examples, Plamper et al. [18] described the synthesis of poly (ethylene oxide) (PEO) / poly-(dimethylaminoethyl methacrylate) (PDMAEMA) miktoarm stars employing Williamson ether synthesis and ATRP polymerization and obtained good systems with thermorresponsive behaviour of copolymer micelles.

One of the most studied hyperbranched systems was bis (Hydroxyl-Methyl) propionic acid polyesters (MPA), which have been used as hyperbranched entities and hyperbranched cores to obtain hyperbranched copolymer macrostructures [2]. These are used in nanomedical applications [19], coatings [20], tumour-targeted molecular imaging probes and therapeutics [21], as biological carriers [22], co-dispersants [23] and more.

Dunjic et al. demonstrated that the rheological properties of aliphatic hyperbranched MPA polyesters are dependent on the pseudogeneration number and nature and degree of modification of the terminal $\mathrm{OH}$ groups in a series. These dependencies are associated with the volume size of shaped and hydrogen bond interactions [5]. In the same line, Adrjanowicz et al. studied the inter- and intra-molecular glass-transition dynamics in MPA hyperbranched polyesters of second, third, and fourth generations. The results indicate that conductivity relaxation becomes increasingly faster than structural relaxation as the glass transition temperature $T g$ is approached, indicating decoupling between translational motions of charges and reorientation of molecules and the hydrogen bonds are crucial in these observations [24]. Andrén et al. [25] functionalized the peripheral hydroxyl groups of MPA hyperbranched polyesters, and a subsequent ATRP procedure of St yielded highly isoporous films with good scaffolding ability.

In a previous work, we prepared hyperbranched copolymers of different architectures and nature of molecular brushes, using atom transfer radical polymerization (ATRP). Nuclear magnetic resonance (NMR) studies allowed to analyse the morphological effect on the stereochemistry of these materials. In addition, thermal and dielectric properties were assessed to investigate the way in which the morphology influenced the dynamics of this group of compounds [26].

The present manuscript is focused on the investigation on the effects of confinement of hyperbranched polymer using simple systems. To prepare the hyperbranched PMPA-PMMA and PMPA-PSt copolymers, we used Bis (HydroxylMethyl) Propionic Acid Polyester (MPA) as the hyperbranched core entity and PMMA and PSt chains as brush shells. To evaluate confinement effects, different generations of hyperbranched MPA were used: generation 2 (16 OH groups, G2), generation $3(32 \mathrm{OH}$ groups, G3) and generation 4 (64 OH groups, G4). NMR studies allowed to determine the composition and average molecular weight of copolymers, as well as the polymer brushes grown from the star-shaped hyperbranched MPA core. The above values were compared to those obtained by size exclusion chromatography. The solution behaviour, size and interactions were evaluated by dynamic light scattering and thermal properties were evaluated through differential scanning calorimetry (DSC) and thermogravimetric analysis (TGA). Our investigations yielded new nanostructured materials based on simple and known systems with architectonic and interesting confinement effect. This is true due to the point that depending on brush nature and arms number, the system changes abruptly its identity properties.

\section{Materials and methods}

\section{Materials}

Hyperbranchedbis - MPA polyester - 64 - hydroxyl, generations $2, \quad 3$ and 4 (97\%, Aldrich), 4-(dimethylamino) pyridine $(99 \%, \quad$ Aldrich), triethylamine (99\%, Sintogran), tetrahydrofuran (RPE, Carlo Erba), 2-bromoisobutyric acid bromide (98\%, Aldrich), methanol (RPE, Anedra), chloroform (RPE, Carlo Erba), dimethylsulfoxide (RA, Anedra), $\mathrm{CuBr}$ (99,995\%, Aldrich), 2,2'-bipyridine (98\%, Biopack), ethyl alpha-bromoisobutyrate (98\%, Aldrich). Monomers, methyl methacrylate (99\%, Aldrich) and styrene (99\%, Aldrich) were freed from the inhibitor by 
washing with aqueous $\mathrm{NaOH}$ solution (10 wt \%) and then with water until neutral, dried over anhydrous sodium sulphate, and distilled under reduced pressure before use.

\section{Hyperbranched copolymers synthesis}

To prepare the hyperbranched PMPA-PMMA and PMPA-PSt copolymers, we used Bis (Hydroxyl-Methyl) Propionic Acid Polyester (MPA) as hyperbranched core and PMMA and PSt chains as brush shells. Hyperbranched MPA generation 2 (16 OH groups, G2), generation 3 (32 OH groups, G3) and generation 4 (64 $\mathrm{OH}$ groups, G4) were employed. $\mathrm{OH}$ terminal groups were acylated with $\alpha$-bromoisobutyryl bromide as described below. After these modifications, the hyperbranched cores were subjected to ATRP polymerization to obtain MMA or PSt shells. ATRP procedures are also described below and illustrated in Scheme 1.

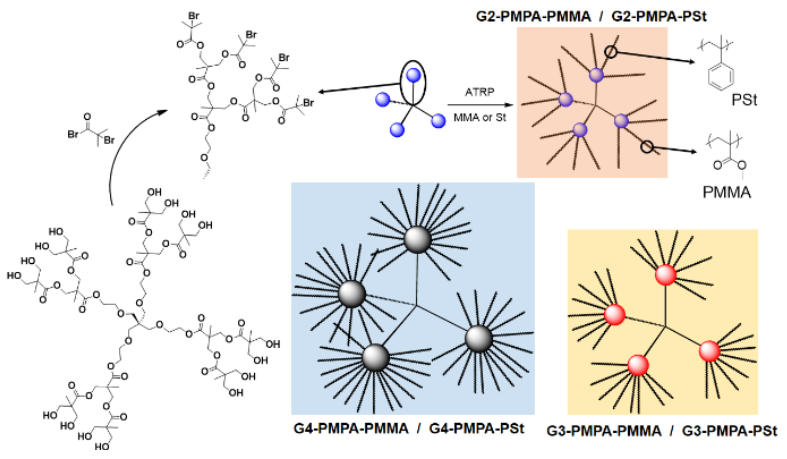

Scheme 1. Schematic hyperbranched copolymers synthesis and structure of all the system obtained.

PMPA-Macroinitiator Cores synthesis.[27] $\alpha$ bromoisobutanoic acid (Bis (Hydroxyl-Methyl) Propionic Acid Polyester) esters were prepared by the reaction of hyperbranched MPA G2, G3 or G4 with $\alpha$ bromoisobutyryl bromide. To achieve this, a solution of $10.0 \mathrm{mmol}$ of hydroxyl groups of hyperbranched MPA (G2, G3 or G4) in $20 \mathrm{ml}$ of dry THF was added to a solution of $16 \mathrm{mmol}$ of 4-(dimethylamino) pyridine and $10 \mathrm{mmol}$ of triethylamine in $7 \mathrm{ml}$ of dry THF under nitrogen atmosphere. Then, $30 \mathrm{mmol}$ of $\alpha$ bromoisobutyryl bromide was added drop-wise at room temperature. After $48 \mathrm{~h}$, a precipitate of 4(dimethylamino) pyridine hydrochloride was filtered off and the solvent containing the macroinitiator evaporated to half volume. The residual solution was precipitated into methanol and the precipitate was dried under vacuum. Yields were 65\% (G2), 59\% (G3) and 68\% (G4).

\section{PMMA and PSt shells synthesis}

- Star-shaped PMPA-PMMA hyperbranched copolymers [26]. The same procedure was carried out for all starshaped PMPA-PMMA hyperbranched copolymers. The amounts of MMA monomer were: 16mmol (G2), 32 mmol (G3) and $64 \mathrm{mmol}(\mathrm{G} 4)$. For each individual experiment, the indicated amount of MMA and 2.66 mmol of bipy in $10 \mathrm{ml}$ of DMSO were placed in a Schlenk flask with a magnetic stirrer and purged with $\mathrm{N}_{2}$ bubbling for 45 minutes. Afterwards, $1.32 \mathrm{mmol}$ of $\mathrm{CuBr}$ was added. Immediately after, the mixture was heated to $75^{\circ} \mathrm{C}$ during 10 minutes with nitrogen bubbling. Then a solution of $1 \mathrm{Br}-\mathrm{mmol}$ of each corresponding PMPAmacroinitiator core was incorporated in $3 \mathrm{ml}$ of DMSO previously purged with $\mathrm{N}_{2}$ bubbling for 10 minutes. After $24 \mathrm{~h}$ of reaction, the reaction mixture was cooled and the catalyst complex was removed by suction filtration through a layer of neutral alumina. The resulting solution was partially evaporated and precipitated into methanol. Each crude star-shaped PMPA-PMMA hyperbranched copolymer was purified by dissolution in chloroform and re-precipitation into methanol.

- Star-shaped PMPA-PSt hyperbranched copolymers. To prepare these copolymers, an adaptation of Angot et al [28] method was employed. As above, the same procedure was performed for all star-shaped PMPA-PSt hyperbranched copolymers. The amount of St monomer were: $16 \mathrm{mmol}(\mathrm{G} 2), 32 \mathrm{mmol}(\mathrm{G} 3)$ and $64 \mathrm{mmol}(\mathrm{G} 4)$. For each individual experiment, the indicated amount of St and $2.66 \mathrm{mmol}$ of bipy (without solvent) were combined in a Schlenk flask with a magnetic stirrer and purged with $\mathrm{N}_{2}$ bubbling for 45 minutes. Afterwards, $1.32 \mathrm{mmol}$ of $\mathrm{CuBr}$ was added. Then, the mixture was heated to $90^{\circ} \mathrm{C}$ for 10 minutes with nitrogen bubbling, and the reaction started with the addition of $1 \mathrm{Br}-\mathrm{mmol}$ of each corresponding PMPA-macroinitiator core. The reaction was performed for 15 minutes and stopped by precipitation into methanol. Each crude star-shaped PMPA-PSt hyperbranched copolymer was purified by dissolution in chloroform, suction filtration through a layer of neutral alumina and re-precipitation into methanol.

\section{Synthesis of linear PMMA and PSt}

Linear PMMA and PSt were obtained using the same methods of star-shaped hyperbranched analogous copolymers, for the linear systems the hyperbranched initiators were replaced for ethyl alphabromoisobutyrate (EBIB). Below the synthetic procedures are explained.

- Linear PMMA. $10 \mathrm{mmol}$ of MMA and $2.66 \mathrm{mmol}$ of bipy in $5 \mathrm{ml}$ of DMSO were placed in a Schlenk flask with a magnetic stirrer and purged with $\mathrm{N}_{2}$ bubbling for 45 minutes. Afterwards, $1.32 \mathrm{mmol}$ of $\mathrm{CuBr}$ was added. Immediately after, the mixture was heated to $75^{\circ} \mathrm{C}$ during 10 minutes with nitrogen bubbling. Then a solution of $1 \mathrm{mmol}$ of EBIB was incorporated in $3 \mathrm{ml}$ of DMSO previously purged with $\mathrm{N}_{2}$ bubbling for 10 minutes. After $24 \mathrm{~h}$ of reaction, the reaction mixture was cooled and the catalyst complex was removed by suction filtration through a layer of neutral alumina. The resulting solution was partially evaporated and precipitated into methanol. Linear PMMA polymer was purified by dissolution in chloroform and reprecipitation into methanol. 
- Linear PSt. $10 \mathrm{mmol}$ of St and $2.66 \mathrm{mmol}$ of bipy (without solvent) were placed in a Schlenk flask with a magnetic stirrer and purged with $\mathrm{N}_{2}$ bubbling for 45 minutes. Afterwards, $1.32 \mathrm{mmol}$ of $\mathrm{CuBr}$ was added and the mixture was heated to $90^{\circ} \mathrm{C}$ for 10 minutes with nitrogen bubbling. The reaction started with the addition of $1 \mathrm{mmol}$ of EBIB. The reaction was performed during 15 minutes and was stopped by precipitation into methanol. The product was purified by dissolution in chloroform, suction filtration through a layer of neutral alumina and re-precipitation into methanol.

\section{Polymers characterization}

The NMR spectra of the polymers were recorded on a Bruker Spectrometer, $300 \mathrm{MHz}$. The typical spectral conditions were as follows: spectral width $3201 \mathrm{~Hz}$, acquisition time $4.09 \mathrm{~s}$ and $8-16$ scans per spectrum. The digital resolution was $0.39 \mathrm{~Hz}$ per point. Chloroform-d1 was the solvent and tetramethylsilane (TMS) the internal standard. The sample concentration was $7.0 \mathrm{wt} \%$.

The average molecular weight and the molecular weight distribution were determined by SEC in a LKB2249 instrument at $25^{\circ} \mathrm{C}$. A series of four $\mu$-Styragel columns $(105,104,103,100$ Å pore size) were used with tetrahydrofuran as eluent. The polymer concentrations were $5 \mathrm{mg} / \mathrm{mL}$, and the flow rate was $0.5 \mathrm{~mL} / \mathrm{min}$. The polymer was detected by infrared (IR) absorption at 5.75 $\mu \mathrm{m}$ with a Miram IA spectrophotometer detector. Poly methyl methacrylate and polystyrene standards supplied by Polymer Laboratories and Polysciences Inc. were used for calibration.

Copolymer thermal properties were evaluated by differential scanning calorimetry (DSC) and thermogravimetric analysis (TGA). DSC measurements were performed using a DSC Q2000 (TA Instruments) under nitrogen atmosphere at $10{ }^{\circ} \mathrm{C} / \mathrm{min}$ heating and cooling rates, from -70 to $150^{\circ} \mathrm{C}$. TGA analyses were performer using a TGA Q500 (TA Instruments). The equipment was kept under a nitrogen atmosphere from room temperature to $700{ }^{\circ} \mathrm{C}$, and a $60 \mathrm{ml} / \mathrm{min}$ gas purge was introduced.

Hydrodynamic diameters and interactions in solution were evaluated by dynamic light scattering (DLS) (Zetasizer Nano Z, laser wavelength $632 \mathrm{~nm}$ ). To determine the scattering intensity of the copolymer dispersion at different copolymer concentrations. The analysis of DLS and size results were carried out through distribution fit. The viscosity values used were pure solvent values at measurement temperatures.

\section{Results and discussion}

\section{Polymer synthesis and characterization}

ATPR polymerization produced hyperbranched polymers whose structure was confirmed by ATR-FTIR and NMR spectroscopies. The characteristic infrared signals were assigned: PMPA-PMMA (G2, G3 and G4): $2950 \mathrm{~cm}^{-1}(\mathrm{C}-\mathrm{H}, \mathrm{Al}) ; 1730 \mathrm{~cm}^{-1}(\mathrm{C}=\mathrm{O}) ; 1460,1390$ and $1370 \mathrm{~cm}^{-1}(\mathrm{C}-\mathrm{H}) ; 1147 \mathrm{~cm}^{-1}$ (C-O). PMPA-PSt (G2, G3 and G4): $3050 \mathrm{~cm}^{-1}$ (C-H aromatic shell); $2950 \mathrm{~cm}^{-1}$ (C-H aliphatic core and shell); $1715 \mathrm{~cm}^{-1}(\mathrm{C}=\mathrm{O}$ core); $1600 \mathrm{~cm}^{-1}(\mathrm{C}=\mathrm{C}$ shell $) ; 730$ and $680 \mathrm{~cm}^{-1}(\mathrm{C}-\mathrm{H}$ monosubstituted aromatic shell).

${ }^{1} \mathrm{H}-\mathrm{NMR}$ analysis allowed not only to identify the copolymers obtained but also to quantify the amount of PMPA core and PMMA and PSt shells (molecular weight determination). ${ }^{1} \mathrm{H}-\mathrm{NMR}$ spectrum and the assignment of resonance signals of G2-PMPA-MI core are shown in Fig. 1A. G2 hyperbranched core-shell structures are illustrated in Figures $I B$ and $I C$ for G2-PMPA-PMMA and G2-PMPA-PSt, respectively.

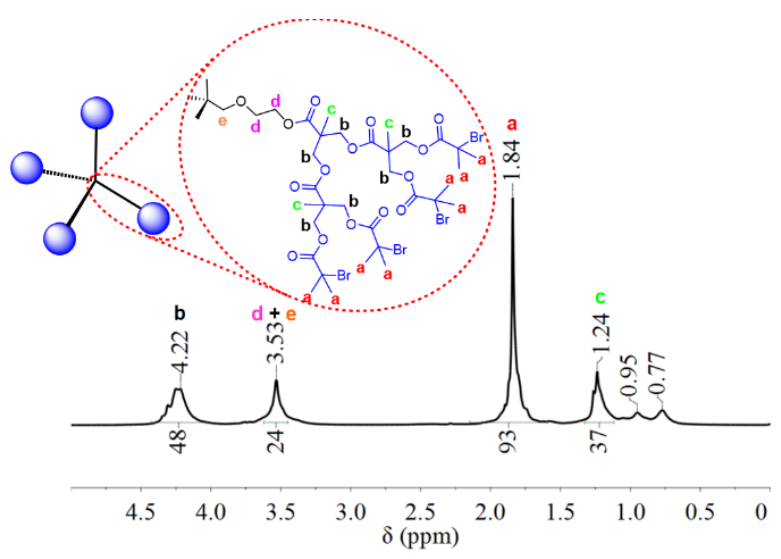

Fig. 1A. H NMR spectrum and assignment of resonance signals for G2-PMPA-MI core.

${ }^{1} \mathrm{H}-\mathrm{NMR}$ spectrum in Fig. 1A is accomplished by a schematic structure of G2-PMPA-MI. The red dotted circle highlights a one-arm chemical structure. The acylation procedure of the hyperbranched G2-PMPA with $\alpha$-bromoisobutyryl bromide replaces all the precursors $\mathrm{OH}$ groups. This was indicated by the absence of $\mathrm{OH}$ signal in ${ }^{1} \mathrm{H}-\mathrm{NMR}$ and was confirmed by the absence of signals around $3000-3500 \mathrm{~cm}^{-1}$ in the IR spectrum. Due to the probabilistic structure and nonrigorous dendritic structure, integrations were normalized to $24 \mathrm{H}$ for signals at $3.53 \mathrm{ppm}$ corresponding to $-\mathrm{CH}_{2}-\mathrm{CH}_{2}$-"d" and - $\mathrm{CH}_{2}$-"e" (6H per arm). Regarding $24 \mathrm{H}$, the signal integration at $4.22 \mathrm{ppm}$ assigned to $-\mathrm{CH}_{2}-$ "b" yielded the expected value of 48 . The signal at $1.24 \mathrm{ppm},-\mathrm{CH}_{3}$ "cc" gave integration for $37 \mathrm{H}$, value close to $36 \mathrm{H}$ expected. The signal at $1.84 \mathrm{ppm}$ confirmed the correct reaction of $\alpha$-bromoisobutyryl bromide with the precursor, as $-\mathrm{CH}_{3}$ groups "a" corresponding to bromoisobutyryl derivate gave integration for $93 \mathrm{H}$ (expected 96H).

Fig. 1B shows the ${ }^{1} \mathrm{H}-\mathrm{NMR}$ spectrum of G2-PMPAPMMA copolymer with a schematic illustration of its star-shaped core-shell hyperbranched structure. The black dotted circle highlights the chemical structure of a PMMA shell brush.

G2-PMPA-PMMA spectrum (Fig. 1B) shows signals of G2-PMPA and PMMA brushes. This coexistence allowed to calculate the relative amount of PMPA and PMMA in the copolymer. In this case, integrations were normalized to $3 \mathrm{H}$ for signals at 
$3.61 \mathrm{ppm}$ corresponding to $-\mathrm{OCH}_{3}$ - "h" of PMMA brush. Signals in the range of $0.8-1.3 \mathrm{ppm}$ correspond to the triad of $\alpha$-methyl signals of PMMA brush. The excess to the expected value for this signal (expected $3 \mathrm{H}$ ) was assigned to the $-\mathrm{CH}_{3}$ "c" " belonging to PMPA core. In the same way, the signal at $1.82 \mathrm{ppm}$ contains $2 \mathrm{H}$ expected from $-\mathrm{CH}_{2}$ " "f" of PMMA brush and $-\mathrm{CH}_{3}$ "a" of PMPA core. The signal at $4.21 \mathrm{ppm}$ assigned to $-\mathrm{CH}_{2}$-" $\mathrm{b}$ " of PMPA integrated for $0.29 \mathrm{H}$ was highlighted in the dotted circle for better visualization. The coexistence of these signals allowed to calculate the average MMA units per brush using Equation 1.

Average MMA units per chain $=\frac{\mathrm{I}_{3.61} / 3 / \mathrm{I}_{4.21} / 48}{16}=10.5$

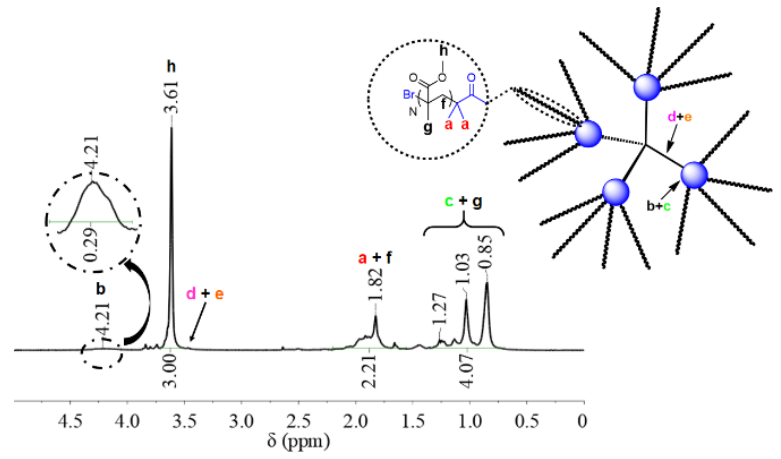

Fig. 1B. 1H NMR spectrum and assignment of resonance signals for G2-PMPA-PMMA core-shell copolymer.

Fig. 1C presents the ${ }^{1} \mathrm{H}-\mathrm{NMR}$ spectrum of $\mathrm{G} 2$ PMPA-PSt copolymer and a schematic representation of its star-shaped core-shell hyperbranched structure with the chemical structure of one PSt shell brush in the black dotted circle.

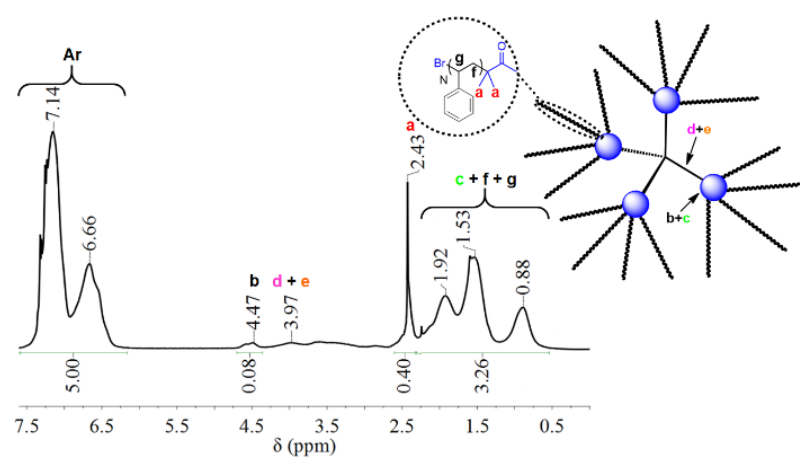

Fig. 1C. 1H NMR spectrum and assignment of resonance signals for G2-PMPA-PSt core-shell copolymer.

In the same way as G2-PMPA-PMMA, Fig. 1C shows signals of G2-PMPA and PSt brushes. For G2PMPA-PSt, the integrations were normalized to $5 \mathrm{H}$ for aromatic signals between 6.4 and 7.6 ppm of PSt brush. Signals in the range of $0.5-2.3$ ppm corresponded to- $\mathrm{CH}_{3}$ "c" belonging to PMPA core overlapping with $-\mathrm{CH}_{2}-$ " $\mathrm{f}$ " and -CH-"g" belonging to PSt shell. The signal at 2.43 ppm was assigned to $-\mathrm{CH}_{3}$ "a" of PMPA integrated for $0.40 \mathrm{H}$. It appears at a higher chemical shift due to the presence of the aromatic shell. The signal at $4.47 \mathrm{ppm}$ assigned to $-\mathrm{CH}_{2}$-"b" of PMPA integrated for $0.08 \mathrm{H}$ also felt the presence of the aromatic shell and appears at a higher chemical shift. Using equation 2 , it was possible to calculate the average St unit per brush.

Average St units per chain $=\frac{\mathrm{I}_{\mathrm{Ar}} / 5 / \mathrm{I}_{2.43} / 93}{16}=15$

The same analysis was carried out for G3 and G4 systems. Table 1 presents the data for all the studied hyperbranched polymers. This table also lists the molecular weight (Mn) calculated via NMR analysis for G2-, G3- and G4-PMPA-MI. Additionally, Mn for all core-shell hyperbranched copolymers was calculated considering the brush number (generation) and brush weight (through average monomer units per chain) and core weight (PMPA-MI). For comparison purposes, along with these values, the Mn and PDI values obtained by size exclusion chromatography (SEC) are also reported. Moreover, Mn and PDI for analogous nonhyperbranched PMMA (G0-PMMA) and PSt (G0-PSt) are presented. In all cases, the Mn obtained by GPC was lower than that calculated by NMR data. Considering that GPC is a relative measurement and that calibrations were performed with a non-hyperbranched standard that these values are comparable to those calculated by NMR. As has been demonstrated by Hirao et al.[29] the Mn vales calculated by NMR are higher than Mn values obtained by GPC, and the differences get bigger as the generation increases.

Table 1. Data for all studied hyperbranched polymers. Average monomer units calculated by NMR, Molecular weight (Mn) calculated via NMR and obtained by GPC.

\begin{tabular}{lcccc}
\hline \multicolumn{1}{c}{ Polymer } & $\begin{array}{c}\text { Average } \\
\text { monomer } \\
\text { units }\end{array}$ & $\begin{array}{c}\mathrm{Mn} \\
(\mathrm{g} / \mathrm{mol}) \\
\text { by NMR }\end{array}$ & $\begin{array}{c}\text { Mn } \\
(\mathrm{g} / \mathrm{mol}) \\
\text { by GPC }\end{array}$ & $\begin{array}{c}\text { PDI } \\
\text { by } \\
\text { GPC }\end{array}$ \\
\hline G2-PMPA-MI & - & 4,088 & 4,097 & 1.6 \\
G3-PMPA-MI & - & 8,328 & 5,251 & 1.5 \\
G4-PMPA-MI & - & 16,808 & 6,484 & 1.8 \\
\hline G2-PMPA-PMMA & 10.5 & 20,888 & 20,036 & 5.9 \\
G3-PMPA-PMMA & 13 & 49,928 & 42,318 & 2.1 \\
G4-PMPA-PMMA & 8.5 & 71,208 & 21,010 & 3.8 \\
\hline G2-PMPA-PSt & 15 & 29,048 & 26,235 & 4.0 \\
G3-PMPA-PSt & 10.5 & 48,264 & 31,869 & 2.1 \\
G4-PMPA-PSt & 11 & 90,024 & 35,684 & 2.2 \\
\hline G0-PMMA & - & - & 2,856 & 1.2 \\
G0-PSt & - & - & 3,052 & 1.2 \\
\hline
\end{tabular}

\section{Thermal evolution}

DSC measurement allowed to establish thermal transitions in star-shaped hyperbranched PMPA-PMMA and PMPA-PSt copolymers. Table 2 lists the $T g$ values for all species, PMPA precursors (G2, G3 and G4), PMPA-macroinitiator cores (G2, G3 and G4) and synthesised star-shaped PMPA-PMMA and PMPA-PSt hyperbranched copolymers (G2, G3 and G4), respectively. For comparison purposes, table 2also give $T g$ values for analogous linear G0-PMMA and G0-PSt. It is worth pointing out that, in all cases, no melting peaks were observed on DSC, thereby indicating the absence of crystalline domain in all polymers. 
Table 2. $T g$ values for all species, PMPA precursors, PMPAmacroinitiator cores, synthesised star-shaped PMPA-PMMA and PMPA-PSt hyperbranched copolymers and linear PMMA and PSt.

\begin{tabular}{lllll}
\hline Polymer & G0 & G2 & G3 & G4 \\
\hline PMPA & & 17.3 & 26.7 & 33.4 \\
PMPA-MI & & 18.1 & 15.6 & 13.9 \\
PMPA-PMMA & 100.5 & 69.2 & 88.5 & 91.9 \\
PMPA-PSt & 101.7 & 81.5 & 53.0 & 57.7 \\
\hline
\end{tabular}

As it can be observed in Table 2, $T g$ values depend on generation levels in star-shaped PMPA hyperbranched precursors and star-shaped PMPA hyperbranched macroinitiators. Star-shaped PMPA hyperbranched showed that $T g$ value increased as polymer generation did, this occurred from $\mathrm{G} 2$ to $\mathrm{G} 3$ and G4. On the other hand, the derivatized samples with $\alpha$-bromoisobutyryl bromide (star-shaped PMPA hyperbranched macroinitiators) displayed the inverse behaviour, $T g$ values increased as generation decreased. As demonstrated by Adrjanowicz et al., the $\mathrm{OH}$ groups in star-shaped PMPA hyperbranched precursors are strongly involved in the dynamic glass transition. These interactions are accumulative, and the result is less segmental mobility of macromolecular chains, less mobility for G4-PMPA after G4-PMPA and finally G2PMPA. On the other hand, PMPA-MI systems do not have $\mathrm{OH}$ free groups, and the interactions are weaker than in the PMPA analogous systems. Therefore, the confinement directly affects the cooperative movements of the polymer chains, and $T g$ values increase from G4PMPA-MI to G3-PMPA-MI and G2-PMPA-MI.

Star-shaped PMPA-PMMA and PMPA-PSt hyperbranched copolymers showed only one-glass transition temperatures, indicative of non-microphase separation in the copolymers. For these systems, an interesting opposite behaviour was observed. In the same way of star-shaped PMPA hyperbranched precursors, PMPA-PMMA $T g$ values increased as polymer generation did, from G2 to G3 and G4, while PMPA-PSt $T g$ values decrease as polymer generation did. These interesting observations indicate different interactions and confinement effects. Glass transition temperature for PMPA-PMMA showed lower mobility/flexibility as polymer generation increase, $T g$ values $69.2^{\circ} \mathrm{C}(\mathrm{G} 2)$, $88.5^{\circ} \mathrm{C}$ (G3) and $91.9^{\circ} \mathrm{C}(\mathrm{G} 4)$. Finally, G4- PMPAPMMA present a $T g$ value lower than linear PMMA (100.5), indicating a confinement effect. On the other hand, PMPA-PSt showed an opposite behaviour; confinement effect is higher for higher generations, showed lower mobility/flexibility as polymer generation decrease, $T g$ values $81.5(\mathrm{G} 2), 53.0$ (G3) and 57.7 (G4). Moreover, G4- PMPA-PSt present a $T g$ value lower than linear PSt, also indicating a confinement effect. The changes in the mobility/flexibility could correspond to different packaging between the core and the shells. In the case of PMMA there is better packaging with the core, however, in the case of PSt, the packaging with the core is hindered and as the generation increases $T g$ value decreases.
Zhang et al. [31] have demonstrated the increase in $T g$ values of star 27 arms PMMA hyperbranched copolymers with long chains shell and the $T g$ values in all cases were higher than $110^{\circ} \mathrm{C}$. The difference with our systems is due to the difference in the chain lengths, our copolymers presented an effect of PMPA core on the values of $T g$, in the cited cases, the large chain length underestimates the effect of the core. On the other hand, Jankova et al.[30] demonstrated that $T g$ values of star 6 arms PSt hyperbranched copolymers depend on the reaction conversion and increase when that value increase but the values in all cases were lower than linear PSt $T g$ value.

Finally, copolymer thermal decomposition was investigated by TGA under a nitrogen stream. Fig. 2A shows the decomposition profile of PMPA precursors (G2, G3 and G4) while figures $2 B$ and $2 C$ present the decomposition profile of the synthesised star-shaped PMPA-PMMA and PMPA-PSt hyperbranched copolymers (G2, G3 and G4), respectively. Also, Figs. 2B and 2C show the analogous linear G0-PMMA and G0-PSt.

The decomposition profile of PMPA precursors (Fig. 2A) exhibited a single thermal event, and the results showed the following stability order G2PMPA $<$ G3-PMPA $<$ G4-PMPA, evidencing higher thermal stability with an increase in generation and molecular weight.

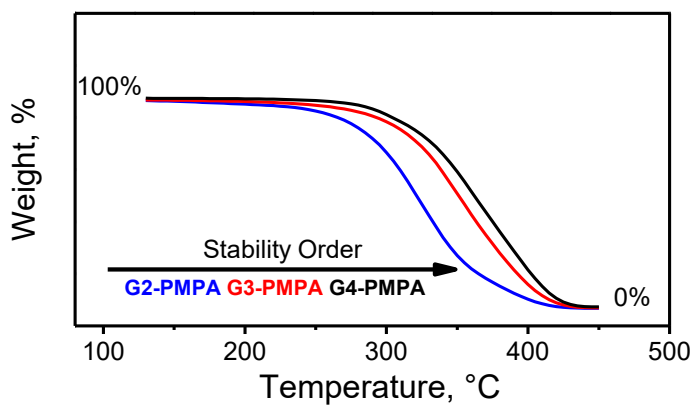

Fig. 2A. Thermal decomposition profile for PMPA precursors (G2, G3 and G4).

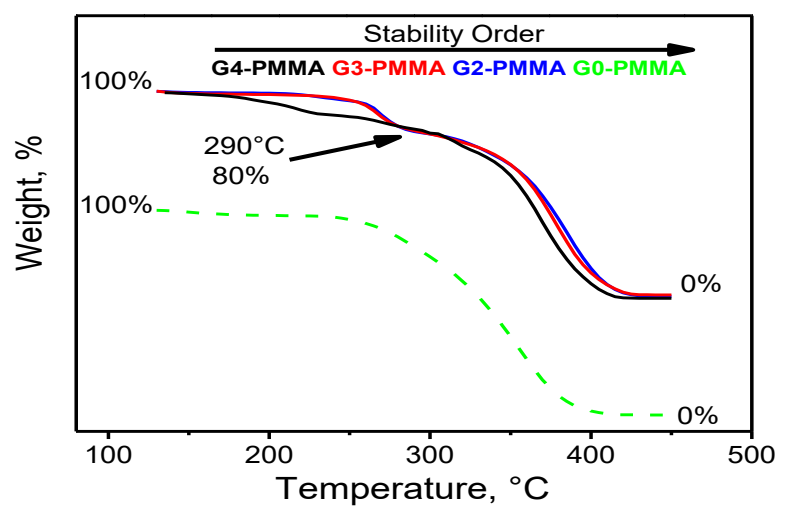

Fig. 2B. Thermal decomposition profile for star-shaped PMPAPMMA hyperbranched copolymers (G2, G3 and G4).

As shown in Fig. 2B, the linear G0-PMMA displays the highest thermal stability; and stability order for the star-shaped core-shell hyperbranched copolymers was 
G4-PMPA-PMMA $<$ G3-PMPA $<$ G2-PMPA-PMMA in opposite direction as displayed by PMPA precursors. The decomposition profile of these systems clearly showed two thermal events for G2- and G3-PMPAPMPA that could be attributed to different fragmentation types in the prepared copolymers. G4-PMPA-PMMA showed more diffuse degradation at the beginning but at approximately $290^{\circ} \mathrm{C}$, the profiles matched and the lost weight at this temperature was close to $20 \%$ for the three samples. This percentage is similar to the percentage weight estimated for the core and shell from Table 1 for each copolymer. In this context, the first thermal event would correspond to degradation of the core structure and the second thermal event to decomposition of the shell brushes.

Star-shaped PMPA-PSt core-shell hyperbranched copolymers showed a diffuse degradation behaviour and the clear degradation event started close to $300^{\circ} \mathrm{C}$ (Fig. 2C). At this temperature, as PMPA-PMMA system, lost weight was approximately $20 \%$ for the three samples.

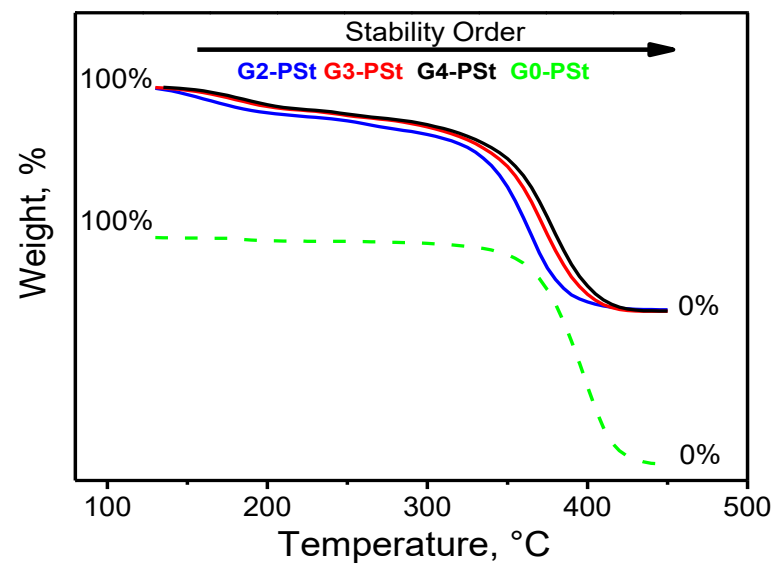

Fig. 2C. Thermal decomposition profile for Thermograms for starshaped PMPA-PSt hyperbranched copolymers (G2, G3 and G4).

\section{Solution properties}

DLS experiments were employed to determine the hydrodynamic diameters $\left(D_{H}\right)$ of synthesised star-shaped PMPA-PMMA and PMPA-PSt hyperbranched copolymers (G2, G3 and G4). Fig. 3 presents the DLS sizes of core-shell systems as a function of generation level.

PMPA-PMMA system showed higher sizes than PMPA-PSt system for all generations. G2-PMPAPMMA and G2-PMPA-PSt showed higher sizes and polydispersity than G3- and G4- analogous. The observed sizes in THF at $10 \mathrm{mg} / \mathrm{mL}$ reveal different interactions between the shell and the solvent. The PSt shells produce a contraction, and in contrast, PMMA shells present more widespread in the solvent in question. G2 systems present bimodal profiles instead of G3 and G4 present monomodal profiles with low polydispersity index.

To evaluate the association between these starshaped MPA-PMMA and MPA-PSt hyperbranched copolymers, Fig. 4 shows the DLS sizes modification for
G4-PMPA-PSt and G4-PMPA-PMMA due to solvent nature changes (THF methanol mixtures, before precipitation, black points 1:1 THF:MeOH and red points 1:3 THF:MeOH). G4-PMPA-PSt showed a clear agglomeration and the particle size increased considerably. In the case of G4-PMPA-PMMA, a clear agglomeration of particles was not observed. These behaviours show different interaction between the systems obtained and the solvents used.
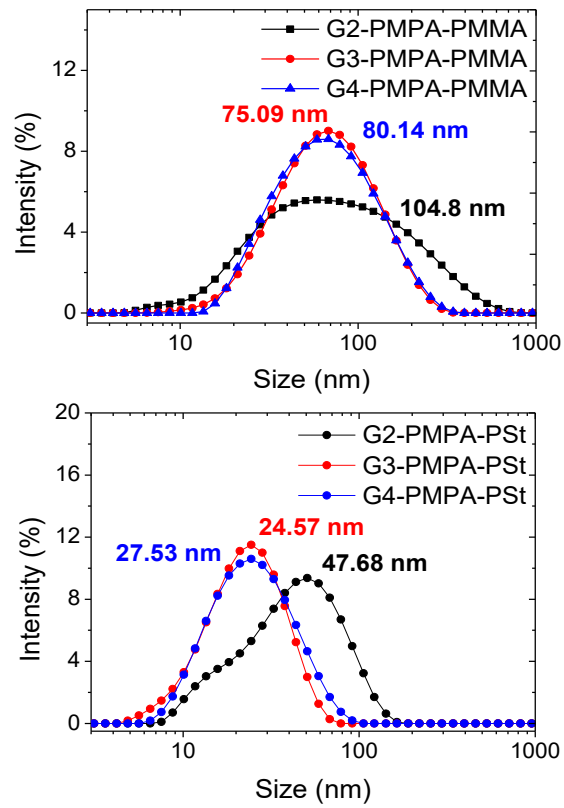

Fig. 3. DLS sizes of core-shell systems in THF $(10 \mathrm{mg} / \mathrm{mL})$ as a function of generation level. DLS results are reported in intensity and analysis was carried out through distribution fit. DLS analysis for G2, G3-, and G4-PMPA-MI gave the following size values: $15 \mathrm{~nm}, 17 \mathrm{~nm}$ and $20 \mathrm{~nm}$ for G2-, G3-, and G4-PMPA-MI, respectively. Sizes for G0PMMA and G0-PSt were lower than $10 \mathrm{~nm}$ in THF solution, $10 \mathrm{mg} / \mathrm{mL}$.
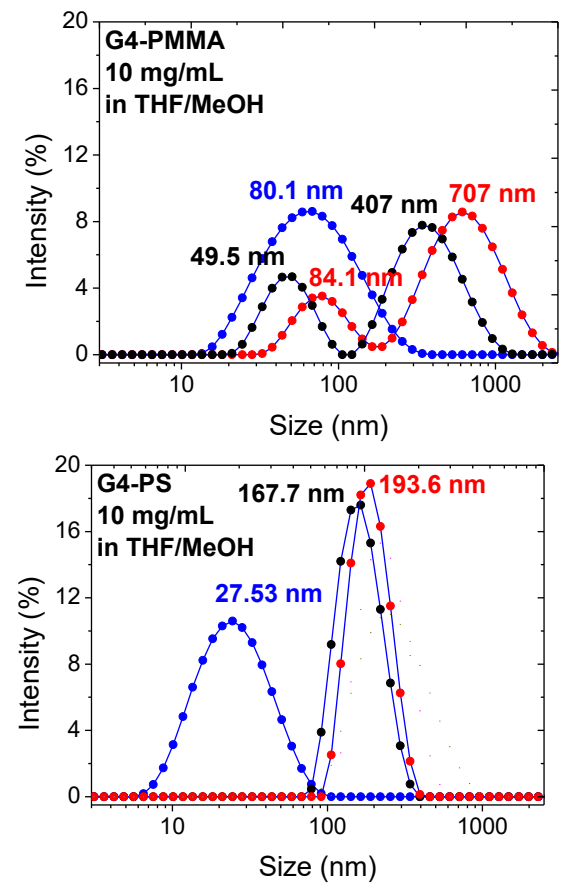

Fig. 4. DLS sizes modification for G4-PMPA-PSt and G4-PMPAPMMA due to solvent nature changes (THF methanol mixtures). 


\section{Conclusions}

Copolymers based on PMPA cores and PMMA or PSt shells have been successfully synthesized using ATRP methods. The obtained copolymers were identified by IR and NMR. Also, by NMR, the average length of the PMMA and PSt chains linked to the core could be estimated, and these values were in all cases around ten monomers.

Molecular weights were estimated by NMR and measured by GPC. For copolymers of generation 2 , the values obtained using these methods were very similar. As generation increased, differences between the values increased, by NMR the molecular weight increased with the increase in generation, while by GCP there were no considerable differences from G2 to G3 and G4. These differences have already been explained in previous works.

Thermal transitions observed in these systems were very interesting and never reported before. Star-shaped PMPA-PMMA and PMPA-PSt hyperbranched copolymers showed only one-glass transition temperatures, indicative of non-microphase separation in the copolymers. In PMPA-PMMA system the $T g$ were in all cases less than linear PMMA, and the $T g$ increased from G2 to G4-PMPA-PMMA. Contrary, for the PMPAPSt system, although also the $T g$ were in all cases less than the linear PSt, the $T g$ decreased from G2 to G4PMPA-PSt. A clear effect of confinement can be seen in our systems and different interactions between the members that form the Star-shaped PMPA-PMMA and PMPA-PSt hyperbranched copolymers. In the case of PMPA-PMMA, the interactions increase from $\mathrm{G} 2$ to $\mathrm{G} 4$, increasing the $T g$, contrary, in PMPA-PSt system, the interactions decrease from G2 to G4, decreasing the $T g$. We propose that these interesting differences are observed due to the low content of monomers contained in the PMMA and PSt shells.

The thermal degradations in both cases showed two degradation events. In which, in a first event lose around $20 \%$ of the mass and in the second $80 \%$. These values are similar to the contents of core and shells that contain the systems studied.

Through DLS, behaviours in solution at concentrations of $10 \mathrm{mg} / \mathrm{mL}$ in THF were studied. The sizes in these conditions were greater for PMPA-PMMA system than for PMPA-PSt system, marking a clear difference in the interactions between the shell and the solvent. The PSt shells produce a contraction, and in contrast, PMMA shells present more widespread in the solvent in question. The addition of a non-solvent for copolymers of generation 4, produced different effects. In G4-PMPA-PSt a clear agglomeration occurred and the particle size increased considerably. In the case of G4PMPA-PMMA, there was a not so clear agglomeration of particles.

The results obtained are relevant to the scientific community because they bring new behaviours observed in hyperbranched systems and can clarify and open new directions for their application.

\section{Acknowledgements}

Authors acknowledge the financial support from CONICET, ANPCyT and UNLP. J.M.G. and M.C are staff members of CONICET, Argentina. G.R.V and G.D. are doctoral and post- doctoral fellows of CONICET.

\section{References}

1. Voit, B. I.; Lederer, A.; Chem. Rev. 2009, 109, 5924

2. Carlmark, A.; Malmström, E.; Malkoch, M.; Chem. Soc. Rev. 2013, 42, 5858 .

3. Gurunathan, T.; Mohanty, S.; Nayak, S. K.; Polym. Plast Technol. Eng. 2016, 55, 92.

4. Froehling, P.; J. Polym. Sci. Part A Polym. Chem. 2004, 42, 3110

5. Dunjic, B.; Tasic, S.; Bozic, B.; Aleksandrovic-Bondzic, V.; Nikolic, M. S.; Djonlagic, J.; J. Appl. Polym. Sci. 2015, 132, 1.

6. Zheng, Y.; Li, S.; Weng, Z.; Gao, C.; Chem. Soc. Rev. 2015, 44 , 4091.

7. Higashihara, T.; Segawa, Y.; Sinananwanich, W.; Ueda, M. Polym. J. 2011, 44, 14.

8. Lu, Y.; Nemoto, T.; Tosaka, M.; Yamago, S.; Nat. Commun 2017, 8 (1), 1863

9. Wang, A.; Xu, H.; Zhou, Q.; Liu, X.; Li, Z.; Gao, R.; Wu, N.; Guo, Y.; Li, H.; Zhang, L.; Electrochim. Acta 2016, 212, 372.

10. Wang, A.; Xu, H.; Liu, X.; Gao, R.; Wang, S.; Zhou, Q.; Chen, J.; Liu, X.; Zhang, L.; Polym. Chem. 2017, 8, 3177.

11. Pal, S.; Hill, M. R.; Sumerlin, B. S.; Polym. Chem. 2015, 6, 7871.

12. Gao, H.; Matyjaszewski, K.; Prog. Polym. Sci. 2009, 34, 317.

13. Nese, A.; Mosnáček, J.; Juhari, A.; Yoon, J. A.; Koynov, K.; Kowalewski, T.; Matyjaszewski, K.; Macromolecules 2010, 43, 1227.

14. Li, W.; Matyjaszewski, K.; J. Am. Chem. Soc. 2009, 131, 10378

15. Gao, H.; Matyjaszewski, K.; Macromolecules 2008, 41, 1118

16. Gao, H.; Matyjaszewski, K.; Macromolecules 2008, 49, 327.

17. Bencherif, S. A.; Gao, H.; Srinivasan, A.; Siegwart, D. J.; Hollinger, J. O.; Washburn, N. R.; Matyjaszewski, K.; Biomacromolecules 2009, 10, 1795.

18. Plamper, F. A.; McKee, J. R.; Laukkanen, A.; Nykänen, A.; Walther, A.; Ruokolainen, J.; Aseyev, V.; Tenhu, H.; Soft Matter 2009, 5, 1812.

19. Alfei, S.; Castellaro, S.; Macromol. Res. 2017, 1.

20. Hadavand, B. S.; Najafi, F.; Saeb, M. R.; Malekian, A.; High Perform. Polym. 2017, 29.

21. McNelles, S. A.; Knight, S. D.; Janzen, N.; Valliant, J. F.; Adronov, A.; Biomacromolecules 2015, 16, 3033.

22. Movellan, J.; González-Pastor, R.; Martín-Duque, P.; Sierra, T.; De La Fuente, J. M.; Serrano, J. L.; Macromol. Biosci. 2015, 15 , 657.

23. Pahovnik, D.; Čusak, A.; Reven, S.; Žagar, E.; J. Polym. Sci. Part A Polym. Chem. 2014, 52, 3292.

24. Adrjanowicz, K.; Kaminski, K.; Dulski, M.; JasiurkowskaDelaporte, M.; Kolodziejczyk, K.; Jarek, M.; Bartkowiak, G.; Hawelek, L.; Jurga, S.; Paluch, M.; Macromolecules 2014, 47, 5798.

25. Andrén, O. C.; Walter, M. V; Yang, T.; Hult, A.; Malkoch, M.; Macromolecules 2013, 46, 3726.

26. Giussi, J. M.; Azzaroni, O.; Hensel-Bielowka, S.; Wojnarowska, Z.; Knapik, J.; Paluch, M.; Polymer (Guildf). 2016, 100, 227.

27. Kreutzer, G.; Ternat, C.; Nguyen, T. Q.; Plummer, C. J. G.; Månson, J. A. E.; Castelletto, V.; Hamley, I. W.; Sun, F.; Sheiko, S. S.; Herrmann, A.; Ouali, L.; Sommer, H.; Fieber, W.; Velazco, M. I.; Klok, H. A.; Macromolecules 2006, 39 (13), 4507.

28. Angot, S.; Murthy, K. S.; Taton, D.; Gnanou, Y.; Macromolecules 1998, 31, 7218 .

29. Hirao, A.; Matsuo, A.; Watanabe, T.; Macromolecules 2005, 38, 8701.

30. Jankova, K.; Bednarek, M.; Hvilsted, S.; J. Polym. Sci. Part A Polym. Chem. 2005, 43, 3748.

31. Zhang, H.; Huang, Z.; Zhang, Q.; Polym. J. 2008, 40, 549. 